\title{
COGNITIVE FUNCTIONING IN PEDIATRIC MULTIPLE SCLEROSIS: TWO YEAR FOLLOW-UP
}

\author{
Kıvılcım Gücüyener', Zeynep Öztürk², Şebnem Soysal', Gökçen Konuşkan², Bahadır Konuşkan³, Asiye Uğraş Dikmen ${ }^{4}, B^{3} a n u ~ A n l a r{ }^{3}$ \\ Gazi University Faculty of Medicine, Department of Pediatric Neurology, Ankara, Turkey' \\ Ankara Children's Hematology-Oncology Training and Research Hospital, Department of Pediatric Neurology, Ankara, Turkey ${ }^{2}$ \\ Hacettepe University Faculty of Medicine, Department of Pediatric Neurology, Ankara, Turkey ${ }^{3}$ \\ Gazi University Faculty of Medicine, Department of Public Health, Ankara, Turkey ${ }^{4}$
}

\section{OBJECTIVE}

Neuropsychological functioning of MS patients with 2 years' interval to evaluate the evolution of the cognitive outcomes and relationships with clinical variables was assessed retrospectively.

\section{METHODS}

MS cases referred to two Turkish MS centers between 2010 and 2016 was included into the study. The extensive neuropsychological battery of the tests non-verbal reasoning, attention/concentration, visuospatial judgement and verbal fluency was used in the current study. Patients were evaluated according to their baseline visit then followed for longitudinal cognitive evaluation two years later. Clinical variables were recorded according to patients' age and sex matched controls were also recruited in the study. Patients were divided according to the age of onset of MS symptoms ( $\leq 12$ years as group I or $>12$ years as group 2 ).

\section{RESULTS}

The study sample consisted of 55 patients with MS and 53 healthy controls. At follow-up, criteria for cognitive impairment (failure on at least 3 tests out of 4 ) was fulfillment in 22 of 46 patients (47\%). EDSS results indicated that patients are associated with cognitive worsening $(0.68 \pm 1.16$ vs $0.04 \pm 0.2, p=0.01)$. In patients who were affected cognitive functions with MS were seen in attention/concentration and non-verbal reasoning, as compared with healthy controls at follow-up. In all patients and group 1 patients, non-verbal reasoning was significantly impaired at follow-up when compared with the initial baseline levels. In group 2 patients, cognitive tests were not impaired at follow-up as compared with the initial baseline assessment.

\begin{tabular}{|l|c|c|c|c|c|}
\hline Test & $n$ & $\begin{array}{c}\text { Baseline } \\
\text { mean } \pm \text { SD }\end{array}$ & $n$ & $\begin{array}{c}\text { Follow-up } \\
\text { mean } \pm \text { SD }\end{array}$ & $p$ \\
\hline RSPMscore & 9 & $30.3 \pm 12.5$ & 9 & $30.6 \pm 13.0$ & 0.829 \\
\hline RSPMtime & 9 & $32.6 \pm 7.7$ & 9 & $37.2 \pm 6.6$ & 0.04 \\
\hline Strooptime & 16 & $43.0 \pm 16.1$ & 16 & $37.7 \pm 16.5$ & $\mathbf{0 . 0 0 3}$ \\
\hline JLOT & 15 & $17.2 \pm 5.3$ & 15 & $18.6 \pm 5.5$ & $\mathbf{0 . 0 3}$ \\
\hline KAS-Animal & 16 & $21.5 \pm 11.8$ & 16 & $21.5 \pm 9.9$ & 0.9 \\
\hline
\end{tabular}

\begin{tabular}{|l|c|c|c|c|c|}
\hline Test & $n$ & $\begin{array}{c}\text { Baseline } \\
\text { mean } \pm \text { SD }\end{array}$ & $n$ & $\begin{array}{c}\text { Follow-up } \\
\text { mean } \pm \text { SD }\end{array}$ & $p$ \\
\hline RSPMscore & 20 & $35.8 \pm 10.9$ & 20 & $38 \pm 13.2$ & 0.1 \\
\hline RSPMtime & 20 & $33.6 \pm 8$ & 20 & $35.5 \pm 7.4$ & 0.1 \\
\hline Strooptime & 30 & $28.3 \pm 9.5$ & 30 & $27.2 \pm 9.3$ & 0.39 \\
\hline JLOT & 29 & $18.7 \pm 5.9$ & 29 & $20.0 \pm 6.1$ & $\mathbf{0 . 0 0 1}$ \\
\hline KAS-Animal & 30 & $27.2 \pm 10.1$ & 30 & $30.7 \pm 13.6$ & $\mathbf{0 . 0 1}$ \\
\hline
\end{tabular}

Table 1. Cognitive findings of patients $\leq 12$ years on the test battery

Table 2. Cognitive findings of patients $>12$ years on the test battery

\begin{tabular}{|l|c|c|c|c|}
\hline Test & $n$ & $\begin{array}{c}\text { Baseline } \\
\text { mean } \pm \text { SD }\end{array}$ & $\begin{array}{c}\text { Follow-up } \\
\text { mean } \pm \text { SD }\end{array}$ & $p$ \\
\hline RSPMscore & 29 & $34.1 \pm 11.5$ & $35.7 \pm 13.3$ & 0.1 \\
\hline RSPMtime & 29 & $33.3 \pm 7.8$ & $36 \pm 7.1$ & $\mathbf{0 . 0 2}$ \\
\hline Strooptime & 46 & $33.4 \pm 13.9$ & $30.9 \pm 13.1$ & 0.01 \\
\hline JLOT & 46 & $18 \pm 5.7$ & $19.3 \pm 5.8$ & $<0.001$ \\
\hline KAS-Animal & 46 & $25.2 \pm 11$ & $27.5 \pm 13.1$ & 0.057 \\
\hline
\end{tabular}

Table 3. Cognitive findings of all patients on the test battery

\begin{tabular}{|l|c|c|c|c|c|}
\hline Test & $n$ & $\begin{array}{c}\text { Patients } \\
\text { mean } \pm \text { SD }\end{array}$ & $n$ & $\begin{array}{c}\text { Controls } \\
\text { mean } \pm \text { SD }\end{array}$ & $p$ \\
\hline RSPMscore & 33 & $36.1 \pm 12.9$ & 53 & $33.3 \pm 8.2$ & 0.2 \\
\hline RSPMtime & 33 & $34.4 \pm 8.3$ & 53 & $27.9 \pm 4.3$ & $<0.0001$ \\
\hline Strooptime & 46 & $30.9 \pm 13.1$ & 53 & $25.6 \pm 2$ & $<0.0001$ \\
\hline JLOT & 46 & $19.3 \pm 5.8$ & 53 & $20.1 \pm 3.4$ & 0.4 \\
\hline
\end{tabular}

Table 4. Cognitive findings of patients and controls on the test battery at follow-up visit

DISCUSSION

Our findings separate from reports on cognitive impairment in children which have shown poor cognitive outcome or inconsistent relationships with disability levels. We confirmed that MS had relatively low impact on cognitive functioning. However, the study showed that, cognitive impairment could be more frequently detected in patients with early onset MS. Cognitive screenings should be performed at early intervention because of the high risk of cognitive difficulties at early onset MS suggests that the importance of effective rehabilitative strategies could prevent negatively affecting school, every day, and social activities of these young patients. 